Chapman University

Chapman University Digital Commons

Psychology Faculty Articles and Research

Psychology

7-2018

Cold Pressor Pain Response in Children with Cancer

Brooke N. Jenkins

Ryan J. Roemer

Ariana Martinez

Tara K. Torres

Michelle A. Fortier

Follow this and additional works at: https://digitalcommons.chapman.edu/psychology_articles

Part of the Oncology Commons, and the Pediatrics Commons 


\section{Cold Pressor Pain Response in Children with Cancer}

\section{Comments}

This is a pre-copy-editing, author-produced PDF of an article accepted for publication in Journal of Pediatric Hematology/Oncology, volume 40, issue 5, in 2018 following peer review. The definitive publisher-authenticated version is available online at https://doi.org/10.1097/MPH.0000000000001219.

\section{Copyright}

Wolters Kluwer Health, Inc. 
Cold Pressor Pain Response in Children with Cancer

Brooke N. Jenkins, ${ }^{1,2}$ PhD; Ryan J. Roemer, ${ }^{2,3}$ PsyD; Ariana Martinez, ${ }^{2,4}$ BS; Tara K. Torres, ${ }^{2,4,5}$

$$
\text { BA; \& Michelle A. Fortier, }{ }^{2,4} \mathrm{PhD}
$$

${ }^{1}$ Department of Psychology, Chapman University

${ }^{2}$ UCI Center on Stress and Health, School of Medicine, University of California-Irvine

${ }^{3}$ Department of Pediatric Psychology, CHOC Children's Hospital, Orange, CA

${ }^{4}$ Department of Anesthesiology \& Perioperative Care, University of California-Irvine

${ }^{5}$ Department of Psychology and Social Behavior, University of California-Irvine

\section{Author Note}

No conflict of interest by any author. This study was funded in part by the UC Cancer Research Coordinating Committee (UCCRCC-53097).

Disclosures: The authors have no conflicts of interest or funding to disclose.

All correspondence concerning this article should be addressed to Michelle A. Fortier, PhD, CHOC Children’s Hospital, 505 S. Main St., Suite 940, Orange, CA 92868, USA, E-mail: mfortier@uci.edu. 


\begin{abstract}
Objective: The goal of this study was to examine pain responses in pediatric patients with cancer. Method: Children (ages 6 to 18) undergoing treatment for cancer $(N=68)$ completed the cold pressor task.

Results: Average pain tolerance was 118.22 seconds $(S D=101.18)$ and $40 \%$ of the children kept their hand in the water the entire 4-minute ceiling. On a 0 to 10 numeric rating scale, children reported a pain severity of $5.07(\mathrm{SD}=3.47)$ at their first report of pain, a pain severity of $5.94(\mathrm{SD}$ $=3.54)$ at their max report of pain, and a pain severity of $5.33(\mathrm{SD}=3.72)$ at the time they reached pain tolerance. Children receiving chemotherapy agents $(N=56)$ with possible neuropathic effects exhibited higher pain tolerance compared to children not receiving such treatments $(N=10), \beta=$ $0.84, S E=0.38$, Wald $X^{2}{ }_{1}=4.88, p=0.027$, hazard ratio $=2.33,95 \%$ confidence interval $(1.10$ 4.92).

Conclusion: This study provides data on experimental pain responses in a sample of children undergoing cancer treatment and suggests that pain experience may be moderated by cancer treatment type.
\end{abstract}




\section{Introduction}

Pain is a complex and multifaceted phenomenon, which can be difficult to assess objectively. Safe and effective methods of experimental pain induction have been used to provide knowledge of pain mechanisms, experience, and responses in controlled settings. ${ }^{1}$ Research involving experimental pain in children has fostered several interventions which minimize pain and improve the management of its negative sequelae in clinical settings. ${ }^{2}$

The cold pressor task (CPT) is one of the most commonly used methods of inducing experimental pain in pediatric populations due to its ethical acceptability and degree of experimental control. ${ }^{3,4}$ The CPT gradually induces ischemic pain through placing a hand or forearm in cold water. ${ }^{5}$ The development and adherence to standardization guidelines for CPT methodology allows for comparison of pain characteristics across populations. ${ }^{4}$ Normative data on pain outcomes for children facilitate standardization of the CPT and may be useful in evaluating the influence of demographic, psychological, and illness-specific variables. For example, Trapanotto et $\mathrm{al}^{5}$ reported that using a 3-minute uninformed ceiling (ie, children did not know they would be asked to remove their hand after 3 minutes) and $10^{\circ}$ Celsius water, $50 \%$ of healthy children between the ages of 8 and 12 years old reached pain tolerance (i.e., removed their hand from the water) within 66 seconds and $35 \%$ of children kept their hands in the water the entire three minutes. Furthermore, consistent with previous research, older children exhibited greater pain tolerance. Previous literature has also shown sex differences in responses to the CPT with male children demonstrating greater pain tolerance. ${ }^{6}$ Normative data such as this but focused on clinical populations may be useful in evaluating how psychological variables such as anxiety and illness-specific variables such as treatment influence pain outcomes. For example, anxiety has been associated with increases in pain perception among healthy children. ${ }^{7}$ It is 
necessary to understand this association between anxiety and pain in clinical populations as well in order to develop tailored interventions.

Although the majority of extant literature on experimental pain has focused on healthy children, the CPT has also been used to study clinical samples of children with chronic illness or recurrent pain, including juvenile rheumatoid arthritis, ${ }^{8,9}$ recurrent abdominal pain, ${ }^{10}$ sickle cell disease, ${ }^{11}$ and headaches ${ }^{12}$ for a review see Birnie et $\mathrm{al}^{2}$ ). Findings in terms of pain behavior differences between clinical and nonclinical pediatric samples have been mixed. For example, 2 studies showed that children with juvenile idiopathic arthritis exhibited lower pain tolerance than controls in response to the $\mathrm{CPT} .{ }^{8,9}$ In contrast, children with recurrent abdominal pain responded with similar pain tolerance and intensity ratings as healthy controls. ${ }^{10}$ Likewise, children with recurrent headaches reported no differences in pain intensity ratings compared to healthy children. ${ }^{13}$ In a study comparing healthy children with a pediatric chronic pain population, no significant overall differences were observed in pain tolerance and pain intensity when an uninformed ceiling was used; however, significant differences emerged in tolerance with a greater percentage of children with chronic pain completing the CPT when an informed, 1 minute ceiling was used (ie, children were aware they would be asked to remove their hand after 1 minute $\left.^{12}\right)$.

Despite the wide body of literature involving the CPT in children, we found no studies involving pediatric samples with cancer, a population who is subject to repeated painful procedures or medical interventions. Evidence suggests that repeated exposure to painful and distressing medical procedures may contribute to the development of chronic pain and impact expectations and reactions to health care interventions and other future painful events. ${ }^{14,15}$ It is therefore likely that children with cancer who must undergo repeated medical procedures may 
have unique responses to experimental pain tasks, such as the CPT. Studying this population may provide valuable information in understanding the developing pain experience of children as well as inform interventions for the management of recurrent pain. ${ }^{3}$ A better understanding of how children with cancer respond to pain could improve clinical practice. For example, altered pain response as a function of exposure to repeated painful procedures or treatment may place children at risk for future chronic pain. ${ }^{16,17}$

The pain resulting from diagnostic and treatment procedures in children with cancer does not decrease with repeated exposure and may worsen in the absence of adequate pain management. ${ }^{18}$ In addition to frequent painful procedures, children being treated for cancer often also suffer from disease-specific pain and side effects of treatment protocols, including mucositis, infection, and chemotherapy-induced peripheral neuropathy (CIPN) ${ }^{19,20} \mathrm{CIPN}$ is an increasingly common neuropathic pain syndrome believed to be caused by neurotoxicity of chemotherapeutic agents that damage the primary sensory neurons. ${ }^{21}$ Painful CIPN symptoms include hyperesthesia; increased physical sensitivity, especially of the skin; allodynia; experience of pain from a nonpainful stimulation such as pressure, heat or cold; and paresthesias, which are abnormal sensations such as tingling or prickling "pins and needles." ${ }^{19}$ In addition, CIPN is also known to cause decreased reflexes and a loss of feeling or sensitivity to temperature and tactile stimuli, which may decrease patients' perception and response to various external stimuli including pain (for review see Gilchrist ${ }^{20}$ ). Thus, there are many factors that may impact the pain experience of children undergoing treatment for cancer. Using the CPT in this population provides a standardized environment to test interventions aimed at reducing pain in children with cancer. The pain children experience during medical procedures can vary dramatically (based on, eg, procedure type, previous experience, or physician skill) and so comparing pain reduction 
strategies using a controlled pain induction technique allows researchers to better assess the direct effects of such pain strategies while controlling for environmental differences. The goal of this paper is to contribute to our body of knowledge on pain experiences of children being treated for cancer in order to improve pain management in this vulnerable population.

Given the dearth of experimental pain research on children with cancer and the importance of understanding factors that impact pain in this population, the purpose of the present study was to investigate pain responses of children undergoing treatment for cancer using the CPT with the following specific aims: (1) descriptively assess pain tolerance and severity as well as explore the correlation between them in children with cancer and (2) examine how demographic, psychological, and treatment-related variables play a role in pain tolerance and severity. On the basis of previous research, ${ }^{5,6}$ specific hypotheses included: (1) greater pain tolerance would be associated with lower pain severity; (2) older children, children who were less anxious, and male children would have a greater pain tolerance and experience less pain severity; and (3) children with treatments that likely lead to CIPN would have higher levels of pain tolerance and lower levels of pain severity.

\section{Materials and Methods}

\section{Participants}

Participants included 68 pediatric patients diagnosed with cancer (38.4\% female), with a mean age of 11.67 years $(S D=3.79$; range $=6$ to 18$)$. Participants were part of a larger study examining the impact of emotion regulation on pain response in children with cancer. Participants were currently undergoing outpatient treatment for cancer at the Hyundai Cancer Institute at the Children's Hospital of Orange County (CHOC Children's) in Southern California. Most (57\%) children were undergoing treatment for leukemias, 14\% were diagnosed with central 
nervous system tumors, $14 \%$ had sarcomas, $7 \%$ had lymphomas, and $8 \%$ had another type of cancer such as germ-cell tumors, Wilms' tumor, and breast cancer. Most (94.5\%) children reported English and 5.5\% reported Spanish as their primary language. Patients excluded from the study were those who (1) had a cognitive impairment, such as a developmental delay that would prevent participation in the CPT, (2) had a medical status requiring inpatient hospitalization or otherwise signified health complications that would prevent participation, (3) had a cut or sore on their non-dominant hand, or (4) had a history of upper extremity vascular disease, high blood pressure, cardiovascular disorder, fainting, seizures, frostbite, or chronic noncancer pain (defined as pain lasting for $>4$ months).

\section{Measures}

Demographic information. Demographic variables including child age, ethnicity, primary language, and sex were collected using self-report measures completed by the child's parent.

Medical Record abstraction. Medical records were abstracted from the CHOC Children's Hospital Hyundai Cancer Institute to assess specific diagnosis and treatment. Treatment type was then coded to reflect whether or not a child was receiving a treatment with a chemotherapy agent known to cause CIPN (CIPN-associated treatment vs. no CIPN-associated treatment).

Pain tolerance. Pain tolerance was defined as the amount of time a child kept their hand in the cold water. ${ }^{4}$ Increased time indicated higher pain tolerance (i.e., that the child could endure more pain).

Numeric Rating Scale (NRS). Children were asked to report their pain and upset at 30 second intervals throughout the $\mathrm{CPT}$, beginning at the first 30 second mark, using numeric rating 
scales with developmentally appropriate anchors. ${ }^{22-24}$ Specifically, children were asked "How much pain are you in (is it hurting) right now?" on a scale of "no pain at all" = 0 to "a lot of pain" $=10$. In addition, children were asked "How upset are you right now?" on a scale of "not upset" $=0$ to "very upset" $=10$. Children also completed the ratings upon removing their hand from the water. First reported pain and upset, worst reported pain and upset (ie, highest value), and reported pain and upset at pain tolerance (ie, time of hand removal) were used as the variables of interest in analyses.

The Childhood Anxiety Sensitivity Index (CASI). The CASI ${ }^{25}$ is an 18 -item self-report scale that measures the tendency to view anxiety-related bodily sensations as dangerous (eg, "It scares me when my heart beats fast"). Items are scored on a 3-point scale (none, some, a lot), and total scores are calculated by summing all items. The CASI has demonstrated high internal consistency $(\alpha=0.87)$ and adequate test-retest reliability (Cronbach's $\alpha$ range $=0.62$ to 0.78 over $2 \mathrm{wk}^{25}$ ) and has been validated with children ages 6 to 18 years old. ${ }^{26-28}$ The reliability of the CASI in this study was high (Cronbach's $\alpha=0.82$ ).

\section{Procedures}

Participants for this study were identified through review of appointment schedules, the participants' medical records, and consultation with the nursing team. Participants were approached on the day of their medical appointment and were asked if they would like to participate. Participants were told that the purpose of the study was to measure their stress response to a difficult task, identified as "The Cold Water Game," in which they would place their hand in cold water for a few minutes and rate how much or little it hurts and how they feel. Families were informed that no lasting risks were involved and they could withdraw from the study at any point without penalty. Informed consent and assent were obtained as appropriate. 
Following consent to participate, participants were scheduled for a study appointment on a day when they were not receiving an infusion, if possible. All procedures were approved by the Institutional Review Board and followed the American Psychological Association Ethical Principles with regard to sample treatment.

On the day of the experiment, participants and their parents were directed to complete demographic information jointly. The child was then separated from their parent to control for parental influence on behavior, and taken to the CPT laboratory room to complete baseline questionnaires and the CPT. The child was instructed "we are going to ask you to place your hand in cold water for a few minutes, but you can remove your hand if it becomes unbearable. While your hand is in the water you will be asked to rate how much or how little it hurts, and how good or how bad you feel at various time points. During the experiment, we will also ask you several questions about your feelings and behavior." As part of the larger study, children were also instructed to engage in one of three emotion regulation strategies (distraction, reappraisal, or empathy) described below.

Emotion regulation strategies. Children were randomly assigned to one of three emotion regulation strategies. In the distraction condition, children viewed a documentary of animals and were told to focus on the video. In the reappraisal condition, children were asked to think about how their participation would help kids like them with cancer. In the empathy condition, the research assistant told the children "Look, I'm really sorry that you have to do this. I know how it feels. The cold can be painful, but don't be nervous. I've been through this and it's alright, you're going to be okay." No group differences in pain emerged between the groups (presented in the results section) and therefore groups were combined for all other analyses. 
Cold pressor task. The CPT consisted of 2 phases, following guidelines suggested by von Baeyer et al. ${ }^{4}$ An experimenter was present throughout the session and stood behind the child during the CPT.

The initial phase, lasting 2 minutes, was conducted with room temperature water in a separate tub to allow the child to become familiarized with the laboratory environment, standardize hand temperature for the CPT, and ensure comprehension of experimental procedures. The child's hand was placed in the water up to the wrist. Every 30 seconds, the experimenter asked the child to provide pain and upset ratings. After the 2-minute baseline, the child was prompted to remove their hand from the tub and provided with a towel to dry their hand.

The child was then informed they would participate in the second phase, the CPT. In the task, the child would place their hand in the cold pressor apparatus, which consisted of a Coleman cooler that was kept cold with a commercial chiller. The cooler had a thermostat and a water pump circulating the water to maintain a temperature of $7^{\circ} \mathrm{C}$. Participants were instructed to submerse their non-dominant hand into the cold water up to their wrist. During this phase, pain and upset ratings were elicited every 30 seconds, up to 8 times during the task, and at the time of hand removal. The experimenter recorded the time of hand removal and the child was directed to complete post-task questionnaires. A 4-minute uninformed ceiling was used such that participants were asked to remove their hand from the water if after 4 minutes they had not already done so. The duration of experienced pain was under the control of the child as they could remove their hand from water at any time, with pain rapidly dissipating subsequent to hand withdrawal.

\section{Statistical Analysis}


First, emotion regulation differences in pain tolerance and self-reported pain and upset were tested. A Cox hazard regression was used to examine emotion regulation condition differences in pain tolerance. Cox hazard regression does not assume a normal distribution for the dependent variable (in this case time until hand removal). Furthermore, because some children did not remove their hand until they were instructed to do so, we right-censored their data as it is unknown when they would have taken their hand out had they not been instructed to do so. Analysis of variance was used to examine emotion regulation condition differences in pain and upset at the three time points of interest (first reported pain and upset, worst reported pain and upset, and reported pain and upset at pain tolerance).

Second, means, SDs, medians, and interquartile ranges were used to calculate averages for pain tolerance and self-reported pain and upset ratings. Pearson product-moment correlations were used to examine relationships between pain, upset, and pain tolerance. Third, pearson product-moment correlations were used to examine how age, sex, and anxiety sensitivity were associated with pain tolerance and self-reported pain and upset. Fourth, a Cox hazard regression model was used to examine group differences between children receiving CIPN-associated treatments versus those taking no CIPN-associated treatments and pain tolerance.

A post hoc power analysis conducted using the power Cox program in STATA 15 revealed that a sample size of 68 would provide $92 \%$ power to detect a large hazard ratio effect with an $\alpha$ of $0.05 .{ }^{29}$ A post hoc power analysis suggested that 68 participants would provide $82 \%$ power to detect a medium effect size correlation $(r=0.30)$ with an $\alpha$ of $0.05 .^{30}$

\section{Results}

\section{Emotion Regulation Group Differences}


When controlling for child age and sex, there were no emotion regulation group differences in pain tolerance, Wald $\chi^{2}{ }_{2}=1.38, P=0.502$. There were no emotion regulation group differences in pain (first reported pain: $F_{2,64}=2.14, P=0.126$; worst reported pain: $F_{2,64}=$ 2.14, $P=0.127$; reported pain at pain tolerance: $\mathrm{F}_{2,64}=1.67, P=0.197$ ) or upset (first reported upset: $F_{2,47}=1.68, P=0.194$; worst reported upset: $F_{2,64}=1.39, P=0.257$; reported upset at pain tolerance: $F_{2,64}=0.86, p=0.429$ ).

\section{Pain Tolerance and Self-Report Pain and Upset}

On average, children exhibited pain tolerance of 118.22 seconds $(\mathrm{SD}=101.18$; median $=$ 67.00; interquartile range [IQR]: 26.25 to $240.00 \mathrm{~s}$ ). A bimodal distribution was present with $60 \%$ of the participants removing their hand on or before the 105 second mark (ie, $1.75 \mathrm{~min}$ ) whereas the other $40 \%$ kept their hand in the water the entire 4-minute ceiling. On average, children reported a pain severity of $5.07(\mathrm{SD}=3.47$; median $=5.00$; IQR: 2.00 to 9.00$)$ at their first report of pain, a pain severity of $5.94(\mathrm{SD}=3.54$; median $=6.00$; IQR: 3.00 to 10.00$)$ at their max report of pain, and a pain severity of $5.33(\mathrm{SD}=3.72$; median $=5.00$; IQR: 2.00 to 9.00$)$ at the time they reached pain tolerance (ie, removed their hand from the water). Children reported an average upset rating of $3.09(\mathrm{SD}=3.82$; median $=1.00$; IQR: 0.00 to 6.00$)$ at their first report of upset, an upset rating of $3.79(\mathrm{SD}=3.95$; median $=2.00$; IQR: 0.00 to 8.00$)$ at their $\max$ report of upset, and $3.22(\mathrm{SD}=3.85$; median $=2.00 ; I Q R: 0.00$ to 6.00$)$ at the time they reached pain tolerance. Child pain and upset were positively correlated, $r_{67}=0.71, P<0.001$. Further, greater pain tolerance was associated with lower pain at first reported pain $\left(r_{65}=-0.48, P<\right.$ $0.001)$, marginally associated with lower pain at worst reported pain $\left(r_{65}=-0.23, P=0.070\right)$, and associated with lower pain at pain tolerance $\left(r_{65}=-0.37, P=0.002\right)$. Similarly, greater pain tolerance was associated with lower upset at first reported upset $\left(r_{65}=-0.45, P<0.001\right)$, worst 
reported upset $\left(r_{65}=-0.33, P=0.007\right)$, and reported upset at pain tolerance $\left(r_{65}=-0.41, P=\right.$ $0.001)$.

\section{Factors Associated with Child Pain Tolerance and Self-Reported Pain and Upset}

Children who were older were more likely to demonstrate higher pain tolerance, $r_{68}=$ $0.27, P=0.027$. Children who were older also tended to report less pain severity (first reported pain: $r_{67}=-0.26, P=0.032$; worst reported pain [marginal]: $r_{67}=-0.22, P=0.075$; reported pain at pain tolerance [marginal]: $r_{67}=-0.24, P=0.051$ ) and upset (first reported upset [marginal]: $r_{67}$ $=-0.23, P=0.061$; worst reported upset: $r_{67}=-0.28, P=0.022$; reported upset at pain tolerance [non-significant]: $r_{67}=-0.18, P=0.142$ ). Sex was not associated with pain tolerance or selfreported pain and upset, $P$ 's $>0.05$. Higher anxiety sensitivity tended to be associated with higher pain (first reported pain: $r_{56}=0.30, P=0.024$; worst reported pain: $r_{56}=0.33, P=0.015$; reported pain at pain tolerance [marginal]: $r_{56}=0.262, P=0.051$ ) and higher average upset (first reported upset [marginal]: $r_{67}=-0.23, P=0.061$; worst reported upset: $r_{67}=-0.28, P=0.022$; reported upset at pain tolerance [nonsignificant]: $r_{67}=-0.18, P=0.142$ ). However, there was no relationship between child anxiety sensitivity and pain tolerance, $r_{57}=-0.16, p=0.236$.

\section{Treatment Type}

Some children $(\mathrm{N}=56)$ were receiving chemotherapy agents known to cause CIPN (eg, vincristine, cisplatin, and bortezomib) while others $(\mathrm{N}=10)$ were not ( 2 children could not be placed into one of the 2 groups and were therefore excluded from analyses using this variable). ${ }^{31}$ There were no significant differences in age, sex, or anxiety between the 2 groups $(P$ 's $>0.05)$. When controlling for child age and sex, children taking CIPN-associated treatments exhibited higher pain tolerance (Fig. 1), $\beta=0.84, \mathrm{SE}=0.38$, Wald $X^{2}{ }_{1}=4.88, P=0.027, \mathrm{HR}=2.33,95 \%$

\footnotetext{
${ }^{1}$ Two children could not be placed into one of the two groups and were therefore excluded from analyses using this variable.
} 
confidence interval (1.10-4.92). Specifically, the hazard rate of hand removal of a child who was not taking an agent associated with CIPN was 2.33 times greater than of a child receiving such a medication. Further, of the 56 children receiving CIPN-associated treatment, $46 \%$ kept their hand in the water for the entire 4-minute period while only $10 \%$ of the children not taking such agents kept their hand in the water for the entire time. Given that anxiety sensitivity and selfreported pain and upset were also associated with pain tolerance, these variables were controlled for (along with child age and sex), in a second Cox hazard model. In this model, children taking CIPN-associated treatments again exhibited higher pain tolerance, $\beta=1.05, \mathrm{SE}=0.48$, Wald $X^{2}{ }_{1}$ $=4.81, P=0.028, \mathrm{HR}=2.84,95 \%$ confidence interval $(1.12-7.23)$.

\section{Discussion}

This study observed the pain response of children undergoing treatment for cancer during the CPT, a widely used experimental pain task in children. Under the conditions of this study, we found children with cancer exhibited average pain tolerance of $\sim 2$ minutes and $40 \%$ of participants kept their hands in the cold water for the entire 4-minute duration of the CPT. Moreover, we determined that several demographic and treatment-related factors impacted pain response, including age, anxiety sensitivity, and chemotherapy agents.

In terms of demographic variables, similar to previous findings in studies of healthy children and clinical populations, ${ }^{5,6,32,33}$ pain tolerance in our sample was higher in older children compared to younger participants. Additionally, older children reported less severe pain and upset throughout the CPT. Children who were more anxious reported marginally more pain and upset but exhibited no difference in pain tolerance compared to less anxious children suggesting that the interrelation between anxiety, pain, and upset did not influence the behavioral outcome of pain tolerance. We did not observe differences in pain tolerance or pain and upset 
severity ratings between male and female participants. Given that findings on the relationship between pain tolerance and sex have been mixed ${ }^{5,6,32,34-36}$ and that they may be age dependent, ${ }^{37}$ further research is needed on potential sex differences in pain in this population. Nevertheless, understanding how demographic variables are associated with pain processing allows for an understanding of how they may influence pain processing over time. For example, if young children or those with greater anxiety sensitivity experience pain, their experience may be more traumatic relative to a much older child or a child with less anxiety sensitivity. This traumatic experience may have greater negative effects on their future perception and anticipation of pain.

In terms of pain response, it can be difficult to compare findings with extant literature due to slight differences in CPT methodology, particularly with regard to water temperature, ceiling length, and whether the ceiling was informed or uninformed. Nonetheless, placing our data on pain tolerance in the context of existing research seems to suggest that our findings are at least consistent with extant literature (e.g., Trapanotto et $\mathrm{al}^{5}$ and Birnie et $\mathrm{al}^{38}$ ) and may even indicate that children undergoing cancer treatment demonstrate increased pain tolerance when compared to healthy children. For example, Myers et a $1^{32}$ employed a sample with a similar age range and water temperature to our sample and their results revealed tolerance at closer to 1 minute, rather than the 2 minutes in the present study. In addition, a large number of children $(40 \%)$ in the current study kept their hands in the cold water for the entire 4-minute ceiling. However, Myers and colleagues' participants also endured multiple pain tasks throughout their experiment and although the other pain task was counter balanced with the CPT, this may have influenced pain tolerance. In addition, Myers and colleagues' instructions to their participants were to keep their hand in the water for "as long as they could" while ours may have alluded to children that they 
only had to keep their hand in for a few minutes making our instructions more similar to those of an informed ceiling.

One explanation for the finding of the high pain tolerance in our sample could be the impact of the cancer treatment children were receiving. In particular, the majority of children were receiving chemotherapy agents known to cause peripheral neuropathy (CIPN). Indeed, children receiving CIPN-associated treatments reported lower pain and upset severity and demonstrated higher pain tolerance compared to children not receiving such treatments. Therefore, it is at least possible that chemotherapy agents may impact children's pain response in a manner that decreases sensitivity to pain. The observation of diminished distress and pain response may be explained by symptoms of sensory and/or autonomic nerve damage or dysfunction, such as hypoalgesia or vasomotor abnormalities. ${ }^{19}$ As mentioned in the introduction, CIPN is known to cause decreased reflexes and a loss of feeling or sensitivity to temperature and tactile stimuli, which may decrease patients' perception and response to various external stimuli including pain (for review see Gilchrist ${ }^{20}$ ). However, although one may hypothesize that decreased pain response could have some benefits, CIPN has negative consequences in terms of sensation (eg, numbness, tingling, burning) that may interfere with procedural pain response but make overall pain management during cancer treatment more complicated. For example, children who have decreased pain sensitivity may be less likely to notice injuries. Interestingly, there were no significant differences in age, sex, or anxiety between children receiving CIPN-associated treatments and those who were not, making it unlikely that these could have explained the group differences in pain tolerance.

In interpreting our data, it is important to be mindful of the fact that our sample size was small and heterogeneous, consisting of children diagnosed with a variety of cancer types and 
undergoing an array of treatment protocols, and therefore may have limited generalizability. This was especially true in our analyses comparing children taking CIPN-associated treatments $(\mathrm{N}=$ 56) and those who were not $(\mathrm{N}=10)$; thus, it is possible results may not replicate in a larger sample size. A second limitation was our categorization of CIPN. Although it was surmised that children receiving CIPN-associated treatments could be experiencing neuropathy, the actual presence and characteristics of neuropathy were not assessed nor was the length of time they had been receiving treatment. As new measures of treatment-induced neuropathies are created and validated, a more detailed understanding of the relationship between CIPN and pain behavior may be observed..$^{39}$ It will be important to explore how stage and length of treatment may influence pain response. Although data on this were not collected, children were likely at difference phases of treatment and therefore frequency and dosing of chemotherapy varied. Furthermore, no healthy comparison group was used in this study. A healthy comparison group would provide a clearer assessment of the differences in pain response to the CPT between children taking chemotherapy agents known to cause CIPN, children with cancer who were not taking such agents, and children without cancer.

An additional limitation of the study was the perceived control as a result of the experimental demands. Children who undergo repeated medical procedures are not provided with the ability to discontinue a painful procedure. Therefore the capacity to end the experimental pain task at their own discretion may have afforded a shift in participant focus, perceived power or predictability during the $\mathrm{CPT}$, consistent with extant literature documenting differences in pain tolerance based upon knowledge of the time-limited nature of the task. ${ }^{12}$ Therefore, knowing that they could withdraw their hand at any time may have influenced their pain perception. Finally, a criticism of the CPT is that it may not relate to real world pain 
experience and this may be particularly relevant in the pediatric oncology population. Pain sensation is comprised of more than simply nociceptive input. There are affective as well as sensory components to the experience of pain. ${ }^{40}$ In children with cancer, these affective components may be particularly salient; a child with cancer who experiences pain may worry that it signals a worsening of the cancer or a procedure may lead to procedural anxiety that may not manifest during an experimental pain task. Thus, it will be important to examine how pain responses to the CPT may predict responses to real world pain for children with cancer.

Children with cancer routinely undergo painful procedures. Therefore, the findings in this study may be useful for future research aiming to mitigate the pain experienced during these procedures and prevent chronic pain in survivorship, given the growing evidence of higher rates of chronic pain in childhood cancer survivors. ${ }^{41}$ Our results suggest that cancer treatment may impact children's pain response. Although more data is needed to confirm this finding, future research would be helpful in identifying how this impacts response to pain during treatment. Future investigations of pain response in this population may also benefit from standardized measures to assess CIPN symptom severity and additional physiological data to further assess the potential impact of chemotherapy agents on children's response to pain. Alternative methods of experimental pain induction may also be considered in evaluating pain behaviors in this population, as well as the use of informed time limits of pain induction. As we learn more about the pain experiences of children with cancer, this body of knowledge can be used to inform pain management approaches in this vulnerable population. 


\section{References}

1. Birnie, K. A., Caes, L., Wilson, A. C., Williams, S. E., \& Chambers, C. T. (2014). A practical guide and perspectives on the use of experimental pain modalities with children and adolescents. Pain Management, 4(2), 97-111. https://doi.org/10.2217/pmt.13.72

2. Birnie, K. A., Petter, M., Boerner, K. E., Noel, M., \& Chambers, C. T. (2012).

Contemporary use of the cold pressor task in pediatric pain research: A systematic review of methods. Journal of Pain, 13(9), 817-826. https://doi.org/10.1016/j.jpain.2012.06.005

3. Birnie, K. A., Noel, M., Chambers, C. T., von Baeyer, C. L., \& Fernandez, C. V. (2011). The cold pressor task: Is it an ethically acceptable pain research method in children? Journal of Pediatric Psychology, 36(10), 1071-1081.

https://doi.org/10.1093/jpepsy/jsq092

4. von Baeyer, C. L., Piira, T., Chambers, C. T., Trapanotto, M., \& Zeltzer, L. K. (2005). Guidelines for the cold pressor task as an experimental pain stimulus for use with children. Journal of Pain, 6(4), 218-227. https://doi.org/10.1016/j.jpain.2005.01.349

5. Trapanotto, M., Pozziani, G., Perissinotto, E., Barbieri, S., Zacchello, F., \& Benini, F. (2009). The cold pressor test for the pediatric population: Refinement of procedures, development of norms, and study of psychological variables. Journal of Pediatric Psychology, 34(7), 749-759. https://doi.org/10.1093/jpepsy/jsn107

6. Schmitz, A.-K., Vierhaus, M., \& Lohaus, A. (2013). Pain tolerance in children and adolescents: sex differences and psychosocial influences on pain threshold and endurance. European Journal of Pain (London, England), 17(1), 124-31. https://doi.org/10.1002/j.1532-2149.2012.00169.

7. Esteve, R., Marquina-Aponte, V., \& Ramirez-Maestre, C. (2014). Postoperative pain in 
children: Association between anxiety sensitivity, pain catastrophizing, and female caregivers' responses to children's pain. Journal of Pain, 15(2), 157-168. https://doi.org/10.1016/j.jpain.2013.10.007

8. Thastum, M., Zachariae, R., \& Herlin, T. (2001). Pain experience and pain coping strategies in children with juvenile idiopathic arthritis. J Rheumatol, 28(5), 1091-1098.

9. Thastum, M., Zachariae, R., Schøler, M., Bjerring, P., \& Herlin, T. (1997). Cold pressor pain: comparing responses of juvenile arthritis patients and their parents. Scandinavian Journal of Rheumatology, 26(4), 272-279. https://doi.org/10.3109/03009749709105316

10. Dufton, L. M., Dunn, M. J., Slosky, L. S., \& Compas, B. E. (2011). Self-reported and laboratory-based responses to stress in children with recurrent pain and anxiety. Journal of Pediatric Psychology, 36(1), 95-105. https://doi.org/10.1093/jpepsy/jsq070

11. Gil, K. M., Edens, J. L., Wilson, J. J., Raezer, L. B., Kinney, T. R., Schultz, W. H., \& Daeschner, C. (1997). Coping strategies and laboratory pain in children with sickle cell disease. Annals of Behavioral Medicine : A Publication of the Society of Behavioral Medicine, 19(1), 22-29.

12. Tsao, J., Evans, S., Seidman, L., \& Zeltzer, L. (2012). Experimental pain responses in children with chronic pain and in healthy children: how do they differ? Pain Research and Management, 17(2), 103-109.

13. Smith, M. S., Martin-Herz, S. P., Womack, W. M., \& McMahon, R. J. (1999). Recurrent headache in adolescents: nonreferred versus clinic population. Headache, 39(9), 616-24.

14. Flor, H., \& Birbaumer, N. (1994). Acquisition of chronic pain: Psychophysiological mechanisms. APS Journal, 3(2), 119-127. https://doi.org/10.1016/S10589139(05)80339-0 
15. Noel, M., Chambers, C. T., McGrath, P. J., Klein, R. M., \& Stewart, S. H. (2012). The influence of children's pain memories on subsequent pain experience. Pain, 153(8), 1563-1572. https://doi.org/10.1016/j.pain.2012.02.020

16. Edwards, R. R. (2005). Individual differences in endogenous pain modulation as a risk factor for chronic pain. Neurology, 65(3), 437-443. https://doi.org/10.1212/01.wnl.0000171862.17301.84

17. Hermann, C., Hohmeister, J., Demirakça, S., Zohsel, K., \& Flor, H. (2006). Long-term alteration of pain sensitivity in school-aged children with early pain experiences. Pain, 125(3), 278-285. https://doi.org/10.1016/j.pain.2006.08.026

18. Hockenberry, M. J., McCarthy, K., Taylor, O., Scarberry, M., Franklin, Q., Louis, C. U., \& Torres, L. (2011). Managing painful procedures in children with cancer. Journal of Pediatric Hematology/oncology: Official Journal of the American Society of Pediatric Hematology/Oncology, 33(2), 119-127. https://doi.org/10.1097/MPH.0b013e3181f46a65

19. Christo, P. J., \& Mazloomdoost, D. (2008). Cancer pain and analgesia. Annals of the New York Academy of Sciences, 1138, 278-298. https://doi.org/10.1196/annals.1414.033

20. Gilchrist, L. (2012). Chemotherapy-induced peripheral neuropathy in pediatric cancer patients. Seminars in Pediatric Neurology, 19(1), 9-17. https://doi.org/10.1016/j.spen.2012.02.011

21. Moore, R. J., \& Groninger, H. (2013). Chemotherapy-Induced Peripheral Neuropathy in Pediatric Cancer Patients. Cureus, 5(6). https://doi.org/10.7759/cureus.124

22. Gragg, R. A., Rapoff, M. A., Danovsky, M. B., Lindsley, C. B., Varni, J. W., Waldron, S. A., \& Bernstein, B. H. (1996). Assessing chronic musculoskeletal pain associated with rheumatic disease: Further validation of the Pediatric Pain Questionnaire. Journal of 
Pediatric Psychology, 21(2), 237-250. Retrieved from

http://www.ncbi.nlm.nih.gov/pubmed/8920155\%5Cnhttp://www.embase.com/search/resu lts?subaction=viewrecord \&from=export\&id=L26112123\%5Cnhttp://dx.doi.org $/ 10.1093 / \mathrm{j}$ pepsy/21.2.237\%5Cnhttp://sfx.library.uu.nl/utrecht?sid=EMBASE\&issn=01468693\&id= doi:10.1093/jpeps

23. Varni, T., Thompson, K., \& Hanson, V. (1987). The Varni/Thompson Pediatric Pain Questionnaire. I. Chronic musculoskeletal pain in juvenile rheumatoid arthritis. Pain, 28, $27-38$.

24. Williamson, A., \& Hoggart, B. (2005). Pain:a review of three commonly used rating scales. Journal of Clinical Nursing, 14(7), 798-804. https://doi.org/10.1111/j.13652702.2005.01121.x

25. Silverman, W. K., Fleisig, W., Rabian, B., \& Peterson, R. a. (1991). Childhood anxiety sensitivity index. Journal of Clinical Child and Adolescent Psychology, 20(2), 162-168.

26. Chorpita, B. F., Albano, A. M., \& Barlow, D. H. (1996). Journal of Clinical Child Psychology Child Anxiety Sensitivity Index : Considerations for Children With Anxiety Disorders Child Anxiety Sensitivity Index : Considerations for Children With Anxiety Disorders. Journal of Clinical C, 25, 77-82.

27. Deacon, B. J., Valentiner, D. P., Gutierrez, P. M., \& Blacker, D. (2002). The anxiety sensitivity index for children : factor structure and relation to panic symptoms in an adolescent sample, 40, 839-852.

28. Weems, C. F., Hammond-laurence, K., Silverman, W. K., Ginsburg, G. S., Weems, C. F., Hammond-, K., ... Golda, S. (1998). Journal of Clinical Child Psychology Testing the Utility of the Anxiety Sensitivity Construct in Children and Adolescents Referred for 
Anxiety Disorders Testing the Utility of the Anxiety Sensitivity Construct in Children and Adolescents Referred for Anxi. Journal of Clinical Child Psychology, 27, 37-41.

29. StataCorp. (2017). Stata Statistical Software: Release 15. College Station, TX: StataCorp LLC.

30. Faul, F., Erdfelder, E., Buchner, A., \& Lang, A. G. (2009). Statistical power analyses using $\mathrm{G}^{*}$ Power 3.1: Tests for correlation and regression analyses. Behavioral Research Methods, 41, 1149-1160.

31. Grisold, W., Cavaletti, G., \& Windebank, A. J. (2012). Peripheral neuropathies from chemotherapeutics and targeted agents: diagnosis, treatment, and prevention. NeuroOncology, 14 Suppl 4(suppl 4), iv45-54. https://doi.org/10.1093/neuonc/nos203

32. Myers, C. D., Tsao, J. C. I., Glover, D. a., Kim, S. C., Turk, N., \& Zeltzer, L. K. (2006). Sex, Gender, and Age: Contributions to Laboratory Pain Responding in Children and Adolescents. Journal of Pain, 7(8), 556-564. https://doi.org/10.1016/j.jpain.2006.01.454

33. Piira, T., Hayes, B., Goodenough, B., \& von Baeyer, C. L. (2006). Effects of attentional direction, age, and coping style on cold-pressor pain in children. Behaviour Research and Therapy, 44(6), 835-848. https://doi.org/10.1016/j.brat.2005.03.013

34. LeBaron, S., Zeltzer, L., \& Fanurik, D. (1989). An investigation of cold pressor pain in children (Part I). Pain, 37(2), 161-171. https://doi.org/10.1016/0304-3959(89)90127-9

35. Tsao, J. C., Glover, D. A., Bursch, B., Ifekwunigwe, M., \& Zeltzer, L. K. (2002). Laboratory pain reactivity and gender: relationship to school nurse visits and school absences. Journal of Developmental and Behavioral Pediatrics : JDBP, 23(4), 217-24.

36. Zeltzer, L. K., Fanurik, D., \& LeBaron, S. (1989). The cold pressor pain paradigm in children: feasibility of an intervention model (Part II). Pain, 37(3), 305-313. 
https://doi.org/10.1016/0304-3959(89)90195-4

37. Boerner, K., Birnie, K., Caes, L., Schinkel, M., \& Chambers, C. (2014). Sex differences in experimental pain among healthy children: A systematic review and meta-analysis. PAIN, 155, 983-993.

38. Birnie, K. A., Parker, J. A., \& Chambers, C. T. (2014). Relevance of Water Temperature, Apparatus, and Age to Children's Pain during the Cold Pressor Task. Pain Practice : The Official Journal of World Institute of Pain. https://doi.org/10.1111/papr.12257

39. Mendoza, T. R., Wang, X. S., Williams, L. A., Shi, Q., Vichaya, E. G., Dougherty, P. M., ... Cleeland, C. S. (2015). Measuring Therapy-Induced Peripheral Neuropathy: Preliminary Development and Validation of the Treatment-Induced Neuropathy Assessment Scale. The Journal of Pain: Official Journal of the American Pain Society, 16(10), 1032-43. https://doi.org/10.1016/j.jpain.2015.07.002

40. Price, D. D. (2000). Psychological and Neural Mechanisms of the Affective Dimension of Pain. Science, 288(5472), 1769-1772. https://doi.org/10.1126/science.288.5472.1769

41. Lu, Q., Krull, K. R., Leisenring, W., Owen, J. E., Kawashima, T., Tsao, J. C. I., ... Zeltzer, L. K. (2011). Pain in long-term adult survivors of childhood cancers and their siblings: A report from the Childhood Cancer Survivor Study. Pain, 152(11), 2616-2624. https://doi.org/10.1016/j.pain.2011.08.006 
Figure 1. Hazard function demonstrating pain tolerance of children taking or not taking agents associated with chemotherapy-induced peripheral neuropathy (CIPN).

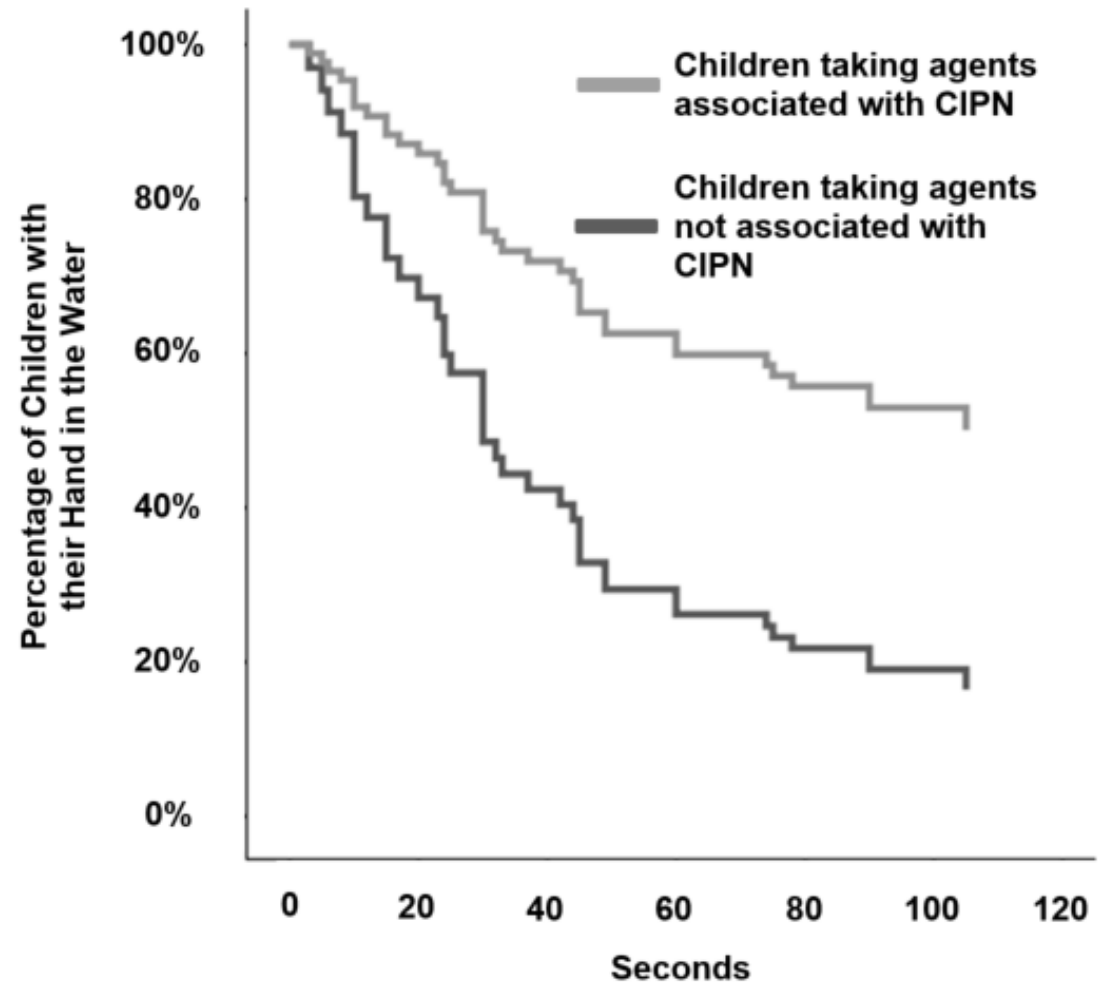

